\title{
Macroscopic diagnostics of microscopic friction phenomena
}

\author{
Sílvio R. Dahmen, ${ }^{1}$ Zénó Farkas, ${ }^{2}$ Haye Hinrichsen, ${ }^{3}$ and Dietrich E. Wolf ${ }^{2}$ \\ ${ }^{1}$ Instituto de Física da UFRGS, Caixa Postal 15051, 90501-970 Porto Alegre RS, Brazil \\ ${ }^{2}$ Department of Physics, Universität Duisburg-Essen, D-47048 Duisburg, Germany \\ ${ }^{3}$ Fakultät für Physik und Astronomie, Universität Würzburg, D-97074 Würzburg, Germany
}

(Received 16 February 2005; published 9 June 2005)

\begin{abstract}
We show that the static friction force which must be overcome to render a sticking contact sliding is reduced if an external torque is also exerted. As a test system we study a planar disk lying on a horizontal flat surface. We perform experiments and compare with analytical results to find that the coupling between static friction force and torque is nontrivial: It is not determined by the Coulomb friction laws alone, instead it depends on the microscopic details of friction. Hence, we conclude that the macroscopic experiment presented here reveals details about the microscopic processes lying behind friction.
\end{abstract}

DOI: 10.1103/PhysRevE.71.066602

PACS number(s): 46.55.+d, 81.40.Pq, 45.70. - n, 81.05.Rm

\section{INTRODUCTION}

Although the scientific investigation of friction started several hundred years ago with the first quantitative experiments by Leonardo da Vinci [1], our knowledge about the microscopic basis for friction is surprisingly incomplete. This applies in particular to the onset of sliding, i.e., the transition from static to dynamic friction. For example, it is an open question whether the contact points give way simultaneously or sequentially on a certain time scale $[2,3]$.

Recently significant progress in this direction was made by Rubinstein et al., who have used fast photo arrays in order to monitor the dynamics of contact points at the onset of sliding [4]. Pushing a Plexiglass slider linearly, they find that the contact points give way in a sequence that travels from the trailing to the leading edge of the block. The front moves initially with half of the surface wave speed, then accelerates, and finally splits up into a sub- and an intersonic front. Although the dynamics of front propagation is not yet fully understood, these experiments show that the time scale on which the contacts give way is very short.

In this paper we study the interplay of translation and rotation at the onset of sliding both experimentally and theoretically. To this end we exert simultaneously a force and a torque on a planar disk lying on a flat surface. Since translational and rotational static friction have the same microscopic origin, they are mutually coupled. In particular, the critical force at the onset of sliding and spinning turns out to depend on the torque, and vice versa. We argue that this critical line of forces and torques, where the disk starts moving, reveals information about the microscopic dynamics, which is not as easily accessible in experiments using linearly moving sliders.

The interplay of force and torque for a sliding disk was studied previously in Refs. [5-7]. It was shown that the sliding friction of a circular disk is reduced if the contact is also spinning with relative angular velocity $\omega-$ a phenomenon that plays an important role in various games such as curling or ice hockey [8-10]. It turns out that this reduction depends on the dimensionless ratio $\varepsilon=v / \omega R$, where $R$ denotes the radius of the disk and $v$ is the tangential relative velocity at the center of the contact area. Based on the Coulomb friction law one obtains a sliding friction force

$$
|\mathbf{F}|=\mu_{d} N \mathcal{F}(\varepsilon)
$$

and a friction torque

$$
|\mathbf{T}|=\mu_{d} N R \mathcal{T}(\varepsilon),
$$

where $\mu_{\mathrm{d}}$ is the dynamic friction coefficient and $N$ is the integrated normal force acting on the contact area. Apart from the limit of pure sliding $\varepsilon \rightarrow \infty$, where $\mathcal{F} \rightarrow 1$, the functions $\mathcal{F}(\varepsilon)$ and $\mathcal{T}(\varepsilon)$ depend on the pressure distribution across the contact area $[5,6,11]$. Assuming uniform pressure over the area of the disk these functions have been evaluated analytically, describing the coupling of force and torque of a circular disk in the sliding case [7].

Turning to static friction let us now consider a resting disk. Applying simultaneously a torque and a force we are interested in the thresholds $\left(\mathbf{F}_{c}, \mathbf{T}_{c}\right)$ at which the disk starts moving. Our daily experience tells us that if we want to move a heavy object across the floor it is easier to do so if we apply a torque to it while pushing. But how are these quantities, force and torque, exactly related? The aim of this paper is to determine this relation experimentally and to study possible theoretical implications with respect to the microscopic aspects of friction.

Regarding the microscopic dynamics, the advantage of friction experiments involving rotational degrees of freedom lies in the fact that stresses at the contact points of the surface with the underlying support are not evenly distributed under simultaneous action of a torque and a force. Therefore, the question arises as to how sliding and spinning set in. Intuitively one may think of two possible scenarios.

(a) When the threshold is reached at those microcontacts where the local stress is maximal, these contacts may break irreversibly. After breaking the released stress is distributed among the remaining microcontacts. As some of these contacts cannot sustain the increasing stress anymore and break, an avalanchelike process sets in so that eventually all contacts break and the whole disk begins to move.

(b) As a different scenario, the broken microcontacts may immediately rearrange themselves to form new contacts, redistributing the released stress over the remaining and the newly formed contact points. This microscopic stick- 


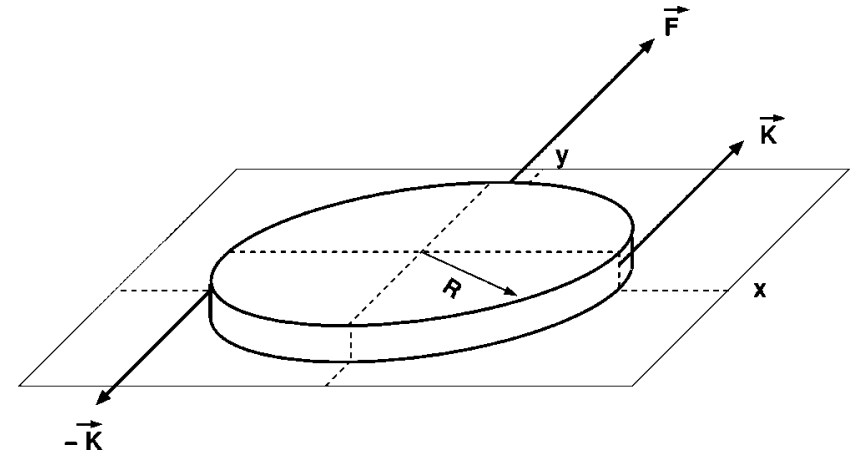

FIG. 1. Schematic view of the experiment: a uniform disk of radius $R$ lies on a flat horizontal surface and is subject to an applied torque $\mathbf{T}=2 R \mathbf{K}$ and force $\mathbf{F}$.

slip creeping continues until all contact points self-organize in such a way that they sustain approximately the same stress. Therefore, by increasing the external force or torque, all microcontacts of a perfectly rigid slider reach the threshold of detachment simultaneously.

We note that experiments such as those of Rubinstein et al. [4], which do not involve rotational degrees of freedom, cannot easily discriminate between the two scenarios. Although the existence of propagating fronts in these experiments seems to favor scenario (a), the high propagation velocity indicates that these fronts may be caused by the inherent elasticity of the slider, leading to slightly higher stresses of the microcontacts at the trailing edge.

In the following section we report on experimental results for a disk subjected to an external force and torque, determining the critical line at which spinning and sliding set in. In Sec. III we study simple microscopic models based on the two scenarios described above in order to calculate the critical line analytically. It turns out that for the avalanche scenario (a) a linear dependence is found while in the second case (b) a nontrivial curve is obtained. Thus the two scenarios lead to a different measurable macroscopic coupling between static force and torque. Comparing these results with the experimental data we can rule out scenario (a) while we find convincing agreement with scenario (b). Finally, in Sec. IV we discuss the dynamics of a disk shortly after the onset of sliding.

\section{EXPERIMENTS}

In order to determine the critical line of detachment we performed a series of experiments where a pulling force and a torque were applied simultaneously to a slider on a horizontal surface.

Most of the experiments were carried out using circular disks made of different materials with radii ranging from $R$ $=149$ to $160 \mathrm{~mm}$ and masses ranging from 324 to $2278 \mathrm{~g}$. All disks had mechanically polished surfaces and were provided with small hooks along the perimeter (see Fig. 1) from which they could be pulled. To measure torques and forces each disk was placed on a fixed and macroscopically flat horizontal surface covered with carpet. Carpet-covered tracks guarantee a more uniform pressure distribution over the contact

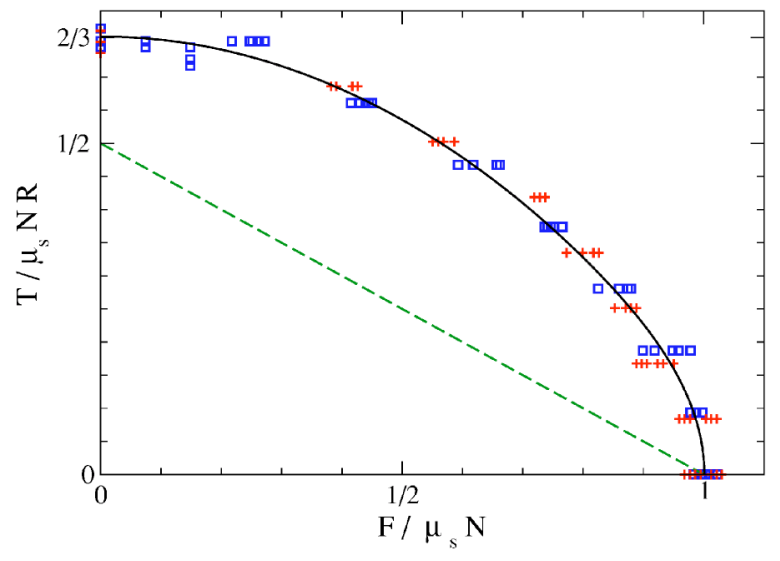

FIG. 2. Measured values of torque and force for a wooden (+) and plastic ( $\square$ ) disk as a function of the dimensionless variables $\mathcal{F}=F / \mu_{s} N$ and $\mathcal{T}=T / \mu_{s} N R$. The dashed and solid lines represent the theoretical predictions for scenarios (a) and (b), respectively (see text for details).

area and have been used successfully in other friction experiments before $[7,12]$. Force meters were then attached to the disks through hooks.

Once the disk was placed on the surface a torque was applied (as indicated in Fig. 1 by the force pair $\mathbf{K}$ and $-\mathbf{K}$ ). The disk was slowly pulled until it started moving. The force meters were set to register the maximum applied pulling force $\mathbf{F}_{c}$. For each fixed value of the torque a set of maximum force readings was made. The experiments were repeated several times under similar temperature and humidity conditions.

In Fig. 2 we present, for the sake of clarity, the results of two selected experiments using disks made of wood and plastic (disks of different materials such as brass or steel with different weights and sizes led to similar results). The curves are parametrized in terms of the dimensionless variables

$$
\mathcal{F}=\frac{|\mathbf{F}|}{\mu_{s} N}, \quad \mathcal{T}=\frac{|\mathbf{T}|}{\mu_{s} N R},
$$

where $N$ is the normal load, and $\mu_{s}$ is the static friction coefficient, which is determined such that the average over the measurements $\langle\mathcal{F}\rangle=1$ at the threshold from sticking to sliding without torque. As can be seen, the experimental results are in excellent agreement with the theoretical prediction for scenario (b), which is shown as a solid line and will be derived in the following section.

For small $\mathcal{F}$ the measurement described above is difficult to perform since the applied torques are already close to their threshold value without additional applied force. Therefore, we inverted the procedure for small $\mathcal{F}$, i.e., forces were kept fixed and torques were varied until the critical threshold was reached. Control experiments confirmed that both types of measurement give compatible results within experimental error.

\section{THEORY}

In order to determine the static thresholds of force and torque analytically for the two scenarios described in the 


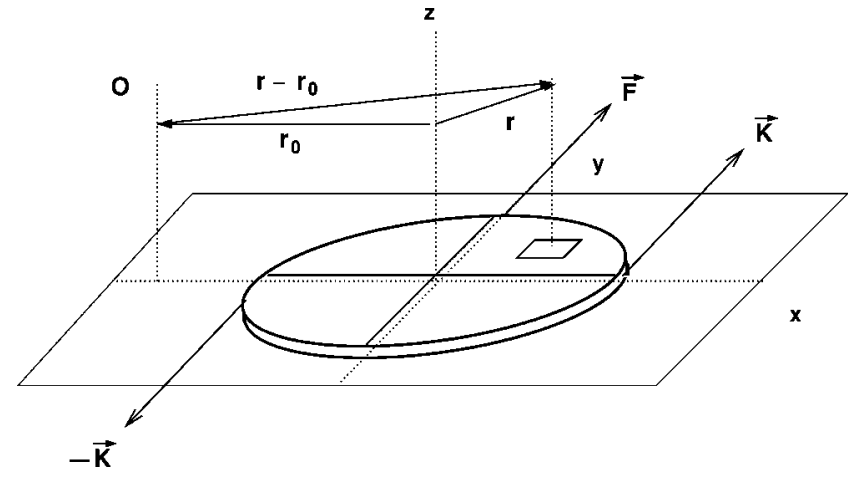

FIG. 3. Geometry used to solve the equations for the coupling of forces and torques. $\mathbf{r}$ is the position of an infinitesimal element of the disk and $\mathbf{r}_{0}$ the position vector of the instantaneous center of rotation $O$.

Introduction, let us consider a simple model in which the microcontacts below the threshold may be thought of as elastic springs. This means that external forces, which are too weak to let the disk slide, are compensated by tiny elastic deformations of microcontacts. These deformations cause a measurable recoil (translation $\delta y$ and rotation $\delta \varphi$ ), when the external forces are switched off. The recoil was observed by placing a small mirror on the surface of the disk and letting a laser beam reflect on it. The beam was projected onto a screen a few meters away so as to make the small displacements visible.

The local displacement of a coarse grained surface element of the disk at a distance $r$ from the center can be expressed by

$$
\mathbf{u}(r, \varphi)=\delta y \mathbf{e}_{y}+r \delta \varphi \mathbf{e}_{\varphi},
$$

where $\delta y$ denotes the displacement of the disk and $\delta \varphi$ is the rotation angle with respect to the center of the disk. We assume that in a coarse grained description the elastic restoring force per unit area is

$$
\mathbf{f}(\mathbf{r})=-k \mathbf{u}(\mathbf{r}) \text {. }
$$

In the case of a circular disk the external force and torque are given by

$$
\begin{gathered}
\mathbf{F}=-\int_{\mathbf{r} \in A} d x d y \mathbf{f}(\mathbf{r}), \\
\mathbf{T}=-\int_{\mathbf{r} \in A} d x d y \mathbf{r} \times \mathbf{f}(\mathbf{r}),
\end{gathered}
$$

where the integrals are performed over the area $A$ of the disk. As long as the slider does not yet move, the local restoring forces integrated over the contact area compensate the external force $\mathbf{F}$ and the external torque $\mathbf{T}$.

We recall that combined translation and rotation of a rigid body in a plane can be interpreted at every moment as a pure rotation around a particular point $\mathbf{r}_{0}=-(\delta y / \delta \varphi) \mathbf{e}_{x}$ (see Fig. 3), so that

$$
\mathbf{u}(\mathbf{r})=\left[\mathbf{e}_{z} \times\left(\mathbf{r}-\mathbf{r}_{0}\right)\right] \delta \varphi .
$$

For later convenience we introduce the dimensionless parameter

$$
\gamma \equiv \frac{r_{0}}{R}=\frac{\delta y}{R \delta \varphi} .
$$

In what follows we assume that a microcontact breaks whenever the local elastic force $|\mathbf{f}(\mathbf{r})|$ exceeds the threshold $\mu_{s} p$, where $\mu_{s}$ is the static friction coefficient and $p$ $=N / \pi R^{2}$ is the normal pressure. It is assumed that the pressure and the friction coefficient are constant throughout the contact area.

(a) First scenario: Breaking of the weakest microcontact. In this case the disk starts sliding as soon as there exists a contact point, $\mathbf{r}$ which exceeds the threshold $|\mathbf{f}(\mathbf{r})|=\mu_{s} p$ triggering an avalanche in which all other points exceed the threshold, too. Obviously the point $\mathbf{r}=R \mathbf{e}_{x}$ at the border of the disk has the largest displacement so that it is the first to reach the threshold. Therefore, the critical rotation angle $\delta \varphi_{c}$ is given by

$$
\delta \varphi_{c}=\frac{\mu_{s} p}{k R(1+\gamma)}
$$

Inserting this result into Eqs. (5) and (8), the force and torque thresholds, Eqs. (6) and (7), become

$$
\begin{gathered}
\mathbf{F}_{c}=\frac{\gamma}{1+\gamma} \mu_{s} N \mathbf{e}_{y}, \\
\mathbf{T}_{c}=\frac{1}{2} \frac{1}{1+\gamma} \mu_{s} N R \mathbf{e}_{z} .
\end{gathered}
$$

The normalized torque threshold $\mathcal{T}=T_{c} / \mu_{s} N R$ is therefore a linear function of the normalized force threshold $\mathcal{F}$ $=F_{c} / \mu_{s} N$ :

$$
\mathcal{T}=\frac{1}{2}(1-\mathcal{F})
$$

(b) Second scenario: Collective breaking of microbonds. The previous result is in marked contrast to the second scenario, where we assume that the forces per unit area, Eq. (5), relax and are redistributed among existing and newly formed microcontacts, thereby self-organizing into a state where virtually all surface elements reach the threshold simultaneously. Since the direction of the displacement in a given point is always the same, we assume that the direction of the local force $\mathbf{u} /|u|$ does not change during this selforganization process; hence

$$
\mathbf{F}_{c}=\mu_{s} p \int_{\mathbf{r} \in A} d x d y \frac{\mathbf{u}(\mathbf{r})}{|\mathbf{u}(\mathbf{r})|},
$$

and

$$
\mathbf{T}_{c}=\mu_{s} p \int_{\mathbf{r} \in A} d x d y \mathbf{r} \times \frac{\mathbf{u}(\mathbf{r})}{|\mathbf{u}(\mathbf{r})|} .
$$

Together with Eq. (4) these integrals are exactly the same as in the sliding and spinning case [7]. One may write them in 


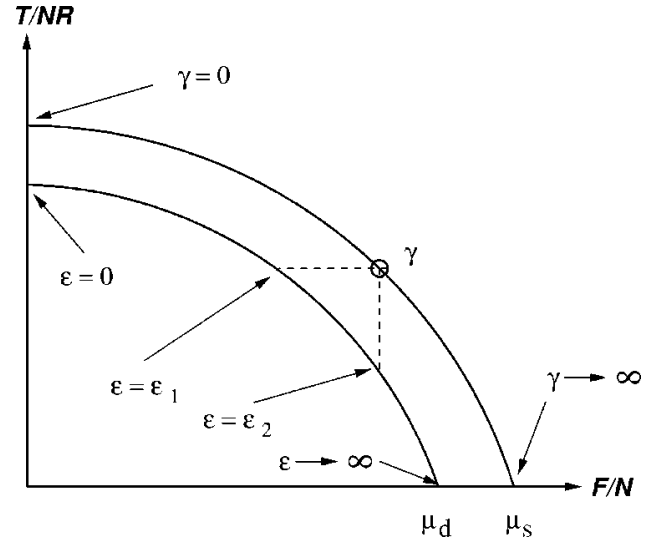

FIG. 4. Schematic plot of (normalized) friction force and torque. The upper curve marks the threshold, below which a static contact is maintained. The lower curve respresents the sliding case. See text for details.

a more transparent way in terms of the previously defined angle $\varphi$,

$$
\mathbf{F}_{c}=\mu_{s} p \mathbf{e}_{\mathbf{y}} \int_{\mathbf{r} \in A} d x d y \frac{r \cos \varphi-r_{0}}{\sqrt{r^{2}+r_{0}^{2}-2 r r_{0} \cos \varphi}}
$$

and

$$
\mathbf{T}_{c}=\mu_{s} p \mathbf{e}_{\mathbf{z}} \int_{\mathbf{r} \in A} d x d y \frac{r^{2}-r r_{0} \cos \varphi}{\sqrt{r^{2}+r_{0}^{2}-2 r r_{0} \cos \varphi}} .
$$

These integrals can be solved exactly and have been shown to depend on $r_{0}$ only through the dimensionless ratio $\gamma=r_{0} / R$. The results for $\mathcal{F}=F_{c} / \mu_{s} N$ and $\mathcal{T}=T_{c} / \mu_{s} N R$ are

$$
\begin{aligned}
& \mathcal{F}(\gamma)=\frac{2(1+\gamma)}{3 \pi \gamma}\left[\left(1+\gamma^{2}\right) E\left(\frac{2 \gamma^{1 / 2}}{1+\gamma}\right)-(1-\gamma)^{2} K\left(\frac{2 \gamma^{1 / 2}}{1+\gamma}\right)\right], \\
& \mathcal{T}(\gamma)=\frac{4(1+\gamma)}{9 \pi}\left[\left(2-\gamma^{2}\right) E\left(\frac{2 \gamma^{1 / 2}}{1+\gamma}\right)+(1-\gamma)^{2} K\left(\frac{2 \gamma^{1 / 2}}{1+\gamma}\right)\right] .
\end{aligned}
$$

Here $K$ and $E$ are the complete elliptic integrals of the first and the second kind, respectively [13]. Although expressed in a more compact form, these two formulas coincide exactly with those for sliding friction $[7,14]$. By varying $\gamma$ between 0 and $\infty$ they provide a parameter representation of the critical curve, which is shown in Fig. 2. Obviously, the curve is in agreement with the experimental data, which indicates the scenario of collective breaking as the physically correct one.

\section{ONSET OF SLIDING}

So far we studied the critical threshold from static to sliding friction. Let us now turn to the dynamics of the disk immediately after the onset of sliding. Figure 4 shows the thresholds for the onset of sliding, $F_{c} / N=\mu_{s} \mathcal{F}(\gamma)$ and $T_{c} / N R=\mu_{s} \mathcal{T}(\gamma)$, which lie on a curve parametrized by the recoil parameter $\gamma=\delta y / R \delta \varphi$. Siding friction and torque, on the other hand, are given by $F / N=\mu_{d} \mathcal{F}(\varepsilon)$ and $T / N R$ $=\mu_{d} \mathcal{T}(\varepsilon)$ with a smaller friction coefficient $\mu_{d}<\mu_{s}$ and the motion parameter

$$
\varepsilon=\frac{v}{\omega R} .
$$

If the force $F_{\text {ext }}$ and torque $T_{\text {ext }}$ reach the threshold for the transition from sticking to sliding at a certain point $\gamma$, the body starts moving, which in general means that it starts sliding and spinning. Hence the question arises, which value of $\varepsilon$ will be selected; in other words, what sliding friction and torque will be observed immediately after the transition from sticking to moving.

The most plausible answer is that $\varepsilon$ will be given by the ratio of velocity and $R$ times the angular velocity an infinitesimal time after the motion started, i.e., Eq. (20) will be replaced by

$$
\varepsilon=\frac{\dot{v}}{\dot{\omega} R} .
$$

The acceleration is given by the difference between static and sliding friction,

$$
m \dot{v}=F_{\text {ext }}-\mu_{d} N \mathcal{F}(\varepsilon)=N\left[\mu_{s} \mathcal{F}(\gamma)-\mu_{d} \mathcal{F}(\varepsilon)\right],
$$

where $m$ is the mass of the slider. Similarly the angular acceleration is given by

$$
\Theta \dot{\omega}=N R\left[\mu_{s} \mathcal{T}(\gamma)-\mu_{d} \mathcal{T}(\varepsilon)\right],
$$

where $\Theta$ is the moment of inertia. Inserting these equations into Eq. (21) one obtains an implicit equation for the value of $\varepsilon$ which will be selected, if the threshold is reached at a given value of $\gamma$ :

$$
\varepsilon=\frac{\Theta}{m R^{2}} \frac{\mu_{s} \mathcal{F}(\gamma)-\mu_{d} \mathcal{F}(\varepsilon)}{\mu_{s} \mathcal{T}(\gamma)-\mu_{d} \mathcal{T}(\varepsilon)} .
$$

It is useful to introduce two special values of $\varepsilon$ for the further discussion, which depend on the point $\gamma$ at which the threshold is reached. $\varepsilon_{1}(\gamma) \in[0, \infty)$ is defined by

$$
\mathcal{T}\left(\varepsilon_{1}\right) \equiv \min \left(\frac{\mu_{s}}{\mu_{d}} \mathcal{T}(\gamma), \mathcal{T}(0)\right) .
$$

Similarly, $\varepsilon_{2}(\gamma) \in[0, \infty)$ is defined by

$$
\mathcal{F}\left(\varepsilon_{2}\right) \equiv \min \left(\frac{\mu_{s}}{\mu_{d}} \mathcal{F}(\gamma), 1\right) .
$$

As $\Theta / m R^{2} \geqslant 0$ and $\varepsilon \geqslant 0$, Eq. (24) implies that $\varepsilon(\gamma)$ is selected from the interval

$$
\varepsilon_{1}(\gamma) \leqslant \varepsilon(\gamma) \leqslant \varepsilon_{2}(\gamma) .
$$

\section{CONCLUSIONS}

In this paper we studied both experimentally and theoretically the coupling between static friction and torque for vari- 
ous disks in dry contact with a track. Our results indicate that before the onset of sliding broken microcontacts between slider and track rearrange themselves to form new contacts, releasing the stresses over the remaining contacts and the newly formed ones. Redistributing the stresses the system self-organizes until all contacts sustain approximately the same stress. Therefore, as the force and torque are increased up to the threshold, all coarse grained surface elements reach their detachment thresholds simultaneously and the slider moves.

Recent experiments by Rubinstein et al. [4] using photoarrays to detect the time evolution of the contact area between a Plexiglass slab and a track of the same material as the threshold is reached indicate that, in the presence of a pushing force only, the process of detachment is accompanied by a series of propagating cracks with three different velocities. The one that propagates most slowly is the dominant mechanism for detachment. These experiments indicate that an avalanchelike detachment scenario takes place at the transition from static to sliding friction, in contradistinction to the previous discussion.

We propose that these results can be reconciled with our findings by considering elastic deformations of the slider. In our experimental setup the disk could be regarded as macroscopically rigid, whereas the Plexiglass slab used in [4] may show local stress building up at the trailing edge when being pushed. This could be checked experimentally by pulling at the leading edge instead of pushing at the trailing one. It would be interesting to see whether the detachment fronts then move in the opposite direction.

We believe that a system under the simultaneous action of a force and a torque represents a favorable experimental setup, since each microcontact is subject to a different displacement, which is not the case when only a force is applied. Therefore it would be interesting to investigate the problem described in this paper with the technique of Rubinstein and co-workers. In particular, how would the propagation of cracks appear in a circular geometry?

The disk geometry we use might seem rather special. However, the concepts presented in this paper can be generalized straightforwardly to other contact geometries as well. An example is given in [15], where a tripod instead of a disk is considered.

\section{ACKNOWLEDGMENTS}

The authors would like to thank E. Magyari and H. Thomas for bringing to their attention the more compact versions of the formulas for $\mathcal{F}$ and $\mathcal{T}$. This project was partially supported by project SFB 445 "Nano-Particles from the Gas Phase" of the DFG.
[1] D. Dowson, History of Tribology (Longman, London, 1979).

[2] M. H. Müser, M. Urbakh, and M. O. Robbins, Adv. Chem. Phys. 126, 187 (2003).

[3] G. V. Dedkov, Phys. Status Solidi A 179, 3 (2000).

[4] S. M. Rubinstein, G. Cohen, and J. Fineberg, Nature (London) 430, 1005 (2004).

[5] S. Goyal, A. Ruina, and J. Papadopoulos, Wear 143, 307 (1991).

[6] R. D. Howe and M. R. Cutkosky, Int. J. Robot. Res. 15, 557 (1996).

[7] Z. Farkas, G. Bartels, T. Unger, and D. E. Wolf, Phys. Rev. Lett. 90, 248302-1 (2003).

[8] T. C. Halsey, Nature (London) 424, 1005 (2003).
[9] K. Voyenli and E. Eriksen, Am. J. Phys. 53, 1149 (1985).

[10] E. T. Jensen and M. R. A. Shegelski, Can. J. Phys. 82, 791 (2004).

[11] M. R. A. Shegelski and R. Holenstein, Can. J. Phys. 80, 141 (2002).

[12] H. Feder and J. Feder, Phys. Rev. Lett. 66, 2669 (1991).

[13] I. S. Gradshteyn and I. M. Ryzhik, Table of Integrals, Series, and Products (Academic Press, New York, 1965).

[14] Note that there is a misprint in the second formula for $\mathcal{T}$ in [7], namely, the parameter $\varepsilon$ should be in the numerator instead of the denominator.

[15] S. R. Dahmen, H. Hinrichsen, A. Lysov, and D. E. Wolf, J. Stat. Mech.: Theory Exp. P033004 (2005). 